\title{
Aplicación del Internet de Las Cosas en el registro automatizado de asistentes
}

\section{The Implementation of the Internet of Things in the automated attendee registration}

\section{ISSN 2071-8748 \\ E-ISSN 2218-3345 \\ (c) (1) $\$$ \\ BY NC SA}

URI: http://hdl.handle.net/11298/982

DOI: https://doi.org/10.5377/entorno.v0i67.7515

\author{
Omar Otoniel Flores-Cortez \\ Universidad Tecnológica de El Salvador omar. \\ flores@utec.edu.sv \\ Verónica Idalia Rosa \\ Universidad Tecnológica de El Salvador veronica. \\ rosa@utec.edu.sv
}

Recibido: 16 de octubre 2018 Aprobado: 11 de marzo 2019

\section{Resumen}

Se denomina internet de las cosas (IoT, siglas del inglés) al área de aplicación de la informática que estudia los conocimientos teóricos y prácticos para el diseño e implementación de dispositivos dotados de sensores y conectividad, permitiendo el fácil control o monitoreo de estado de estos objetos a través del internet. Es dentro de este campo de estudio que se enfocó el presente trabajo, cuyo objetivo principal fue el proponer nuevo conocimiento científico sobre el uso de tecnologías y técnicas del IOT; en la solución de una situación problemática específica: el registro automatizado de los asistentes a un recinto o aula. La investigación realizada propuso el diseño, desarrollo y validación de un sistema IOT de bajo costo, para el registro automatizado vía internet de asistentes, basado en la información recogida por sensores electrónicos inalámbricos dispuestos en la entrada del lugar. El resultado principal de esta investigación fue dividido en diferentes bloques: un circuito electrónico, el firmware para plataforma IoT y la

\section{Abstract}

What the internet of things (IOT) makes reference to is the application field in the computer sciences that studies the theoretical and practical knowledge for the design and implementation of devices equipped with sensors and connectivity, thus allowing for an easy control or status monitoring of these objects through the internet. It is within this field of study that this work is focused; its main objective was to propose new scientific knowledge about the use of IOT technologies and techniques in solving a specific problem situation: the automated attendee registration to a campus or classroom. This research proposed the design, development and validation of a low cost IOT system for automated attendee registration via internet, based on information collected by wireless electronic sensors arranged at the entrance of the site. The main findings of this research were divided in different blocks: an electronic circuit, and the firmware for loT platform plus the display for the user. The scientific knowledge and IOT techniques applied [in this research] 
visualización al usuario. Los conocimientos científicos y las técnicas de IoT aportan gran ayuda en el diseño de soluciones de bajo coste y sus aplicaciones van mucho más allá de la realizada durante esta investigación, siendo un fundamento muy importante en el desarrollo de ambientes inteligentes.

\section{Palabras clave}

Internet de las cosas-tecnología, internet, búsquedas en internet, información tecnológica, administración de sistemas de información.

\section{Introducción}

Una parte muy importante en la vida del hombre siempre ha sido la tecnología, ha usado su inteligencia en la creación de artefactos y herramientas para facilitar sus labores. Hoy en día, las tecnologías de la información y comunicación (TIC) están en todas las áreas del quehacer humano; todos los días interactuamos con ellas, muchas veces sin darnos cuenta. Una de estas es la llamada internet de las cosas.

El internet de las cosas (IOT) es un área tecnológica que refiere al diseño e implementación de dispositivos electrónicos embebidos conectados al internet, tales dispositivos están empotrados dentro de todo tipo de objetos de uso diario, permitiendo el fácil control o monitoreo de estado de estos objetos a través del internet gracias al dispositivo electrónico dentro de ellos (Tollervey, 2017).

En este contexto, se encuentra una subárea de aplicación denominada ciudades inteligentes (smart cities), cuyo fin es aplicar las técnicas del IoT a entornos dentro de edificios.

\section{Planteamiento del problema}

Actualmente el uso de sistemas electrónicos automatizados aplicados en la solución de tareas cotidianas o repetitivas es una necesidad; optimizar procesos y recursos económicos y humanos es prioritario para toda institución aid in designing low-cost solutions. Said applications can go far beyond from what was found during this investigation and are a very important foundation in the development of intelligent environments.

\section{Keywords}

Internet of things-technology, internet, internet searches, technological information, information systems management. moderna. Específicamente, aquellas que ofrecen servicios a grupos de usuarios de forma masiva, tales como escuelas, universidades, salas de eventos o hasta instituciones que poseen una planta de recurso humano grande; se encuentran ante una tarea casi inevitable: el registro de las personas que asisten a la institución, evento, clase, concierto, congreso, charlas, etc. Si enfocamos esta situación en un ambiente académico, específicamente universitario o escolar, se hace evidente que se necesitará el registro de asistencia al aula o aulas, y que dicha tarea es a veces muy compleja de realizar; y más cuando el campus es de gran extensión, tiene muchas aulas y diversos horarios de clases.

Al realizar un recorrido de campo por diferentes universidades y centros escolares del entorno nacional, y hacer una consulta sobre la metodología de registro de asistencia, encontramos que esta tarea actualmente es realizada haciendo un conteo visual y con un registro manuscrito, además, utilizando personal académico para la realización de un recorrido a pie por cada una de las aulas.

En el caso de estudio específico, el de la Universidad Tecnológica de El Salvador (Utec), uno de los procesos de índole administrativo que más demanda tiempo y esfuerzo por parte del cuerpo académico es el conteo de los estudiantes asistentes a las sesiones de clases en los horarios programados. Este es un proceso de recolección que se está desarrollando de forma manual; y en ocasiones también su procesamiento y notificación. 


\section{Justificación}

La investigación aplicada es una forma de lograr soluciones de alto nivel científico en los problemas de la sociedad actual; y se vuelve de suma importancia para los pueblos cuando la implementación permite brindarles beneficios colaterales.

En la actualidad, las instituciones especializadas de servicio están obligadas a mejorar sus procesos internos y de atención al usuario o cliente. Esta responsabilidad implica muchas acciones e implementaciones en varias áreas, desde la administración, la producción hasta servicio al cliente, sean estas mejoras humanas, logísticas, estructurales y técnicas.

El registro de asistencia en un centro educativo permite llevar un mayor control de la población estudiantil, con lo que se pueden sacar ciertos datos estadísticos en la toma de decisiones a corto y largo plazo. Aunado a esto, debido a que la realidad salvadoreña demanda cada día una mayor atención en el área de la seguridad, surge la necesidad de disponer de un mecanismo que proporcione el registro de quienes ingresan a la institución, y además que sea una clara advertencia a personas ajenas a la universidad de que el nivel de monitoreo es permanente y efectivo.

La propuesta viable en coste económico y de rápida implementación, son los sistemas de identificación por radiofrecuencia (RFID, por sus siglas en inglés). Este trabajo propuso un sistema electrónico de IOT económico que se colocó en la entrada del aula, que registra a cada estudiante que ingresa mediante el uso de un carné de RFID, y que además el mismo sistema notifique en tiempo real, vía acceso a un sitio web, información de asistentes a los administradores del centro educativo. Los resultados este trabajo se estiman que podrán ser replicados y de beneficio no solo para la institución estudiada, sino para otras industrias como supermercados, mercados, almacenadoras, importadores, exportadores, etc.

Un sistema para registro de estudiantes con capacidad de notificación por internet, a diferentes personas, cada segmento de horario de clases no existe en el sistema educativo local. Sin embargo, dentro del mercado comercial de El Salvador existen empresas (representantes de marcas) dedicadas a implementar este tipo de soluciones, que a la larga son de costo elevado y no permiten una flexibilidad de adaptación a cada institución, ya que son sistemas cerrados diseñados y construidos por terceros. Cabe destacar que un sistema computarizado de registro de personas con las características planteadas por este trabajo no está disponible en el mercado (Ejje, 2017).

\section{Método}

En el desarrollo de este trabajo se empleó el método experimentación sin hipótesis, el cual es "un método empleado en casos donde la investigación tiene por objeto el provocar determinados fenómenos que no se presentan usualmente en la naturaleza y cuyo conocimiento puede ser interesante o importante en el avance de la ciencia y la tecnología" (Sánchez, 2016). Tomando como base lo anterior y con el objetivo propuesto de diseñar e implementar un sistema IOT para el registro de los asistentes a un aula, el trabajo se desarrolló por etapas, tal como se describe a continuación.

\section{Arquitectura de un sistema de IoT.}

El primer paso para la implementación de un sistema de loT es el diseño de la arquitectura de bloques de la aplicación. Basado en las investigaciones sobre el estado de la técnica, se establecieron las siguientes etapas funcionales para la implementación del sistema que se propondría: 1) Sensores: lectura de magnitudes físicas y conversión de señal. 2) Procesamiento electrónico: normalmente implementado con un microcontrolador, que incluye memoria para almacenamiento del firmware. 3) Conectividad: para la conexión con algún tipo de red alámbrica o inalámbrica para acceder a internet. 4) Plataforma IOT: servicio informático en internet donde se almacenará y procesará la información recibida desde el dispositivo electrónico (Kubitza, 2016). 5) Visualización: servicios usados para el acceso del usuario y visualizar la información producida por los sensores. En la figura 1 se muestra la arquitectura diseñada para este sistema planteado. 
Figura 1. Diseño de la arquitectura del sistema propuesto

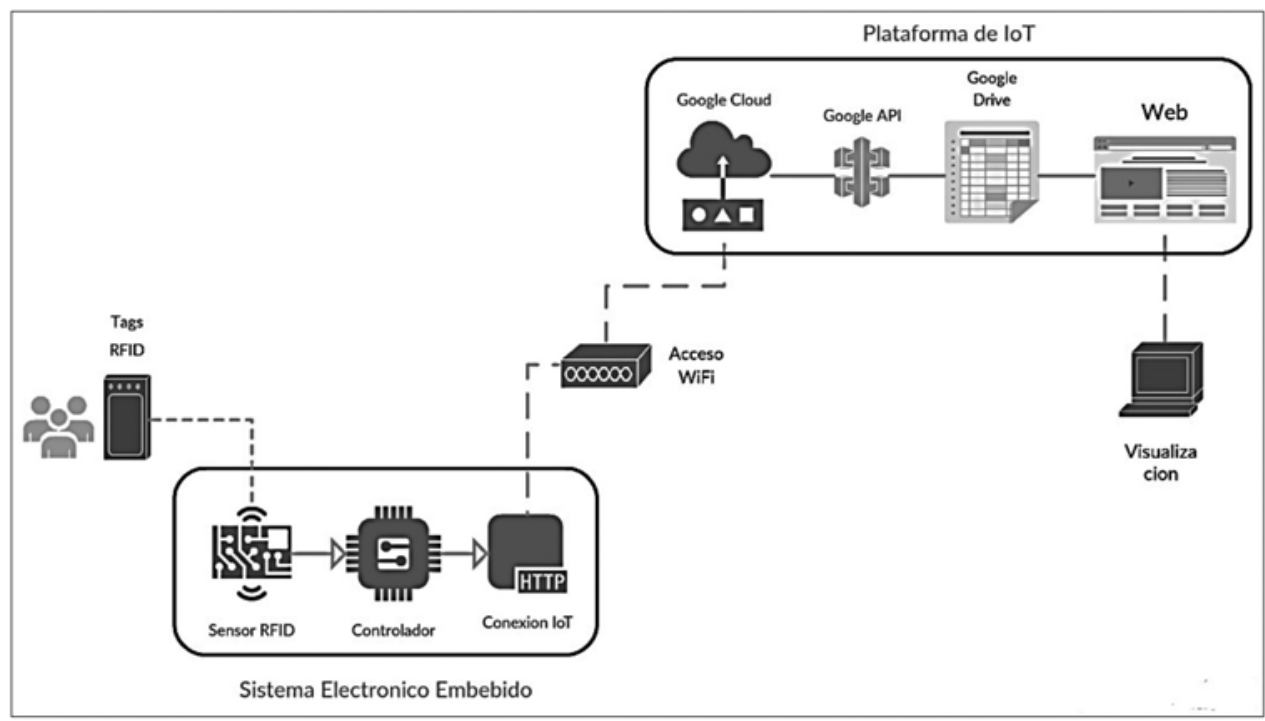

Fuente propia

\section{Selección de componentes del sistema IoT}

A partir de lo anterior y de la información recabada a partir del estado de la técnica, se seleccionaron las siguientes herramientas tecnológicas para la implementación de cada etapa del sistema IOT propuesto: 1) Sensores: se utilizaron dispositivos con tecnología de RFID, específicamente del modelo MFRC522, con frecuencia de trabajo $13.56 \mathrm{MHz}$. A cada estudiante del grupo de prueba se le entregó una tarjeta o tag de RFID, el cual fue grabado previamente con el número de carné correspondiente. 2) Procesamiento electrónico: se utilizó el microcontrolador ESP8266, junto con la placa de desarrollo NodeMCU, para la implementación de todo el procesamiento electrónico y de firmware que gobernará los sensores y el procesamiento de las señales producidas. 3) Conectividad: la placa NodeMCU, además del microcontrolador ESP8266, ya incluye un transceptor WiFi capaz de conectarse a una red inalámbrica disponible en el sitio de implementación. 4) Plataforma IoT y presentación: buscando la mejor opción en coste-beneficio, se usaron los servicios incluidos en la suite de Google para realizar las funciones de este bloque (Mcpherson, 2016).

\section{Diseño de plataforma IoT}

La etapa denominada Plataforma IoT del sistema diseñado se basa en los servicios de Google, específicamente se utilizaron las aplicaciones Google App Script y Google Sheets. Primero se diseñó un código o script que fue almacenado y ejecutado en los servidores, en la nube, de Google. Este es un programa en lenguaje de programación Java que se encarga de recibir, por medio de protocolo HTTP, los datos de la tarjeta del circuito electrónico escaneada proveniente, que a su vez son enviados a una hoja de cálculo en Google Drive, para su almacenamiento y visualización dentro de un sitio web diseñado en la aplicación Google Sites.

\section{Resultados}

\section{Circuito electrónico embebido}

Se diseñó un circuito electrónico de conexión para el sistema embebido encargado de capturar la información del carné de RFID escaneado, que se basa en la plataforma de desarrollo NodeMCU y en el microcontrolador ESP8266, lo cual permite un diseño minimalista pero técnicamente eficiente. En la figura 2, se puede apreciar el circuito diseñado para la etapa de captura, procesamiento y envío de datos a internet. El circuito electrónico fue programado usando un firmware, usando lenguaje de programación $\mathrm{C}$ y basado en el algoritmo básico pero eficaz de cuatro funciones: captura, procesamiento, conexión y envío a Google App Script, esto cada vez que se presenta una nueva tarjeta RFID. En la figura 3 se observa el circuito implementado. 
Figura 2. Diseño del circuito electrónico embebido propuesto

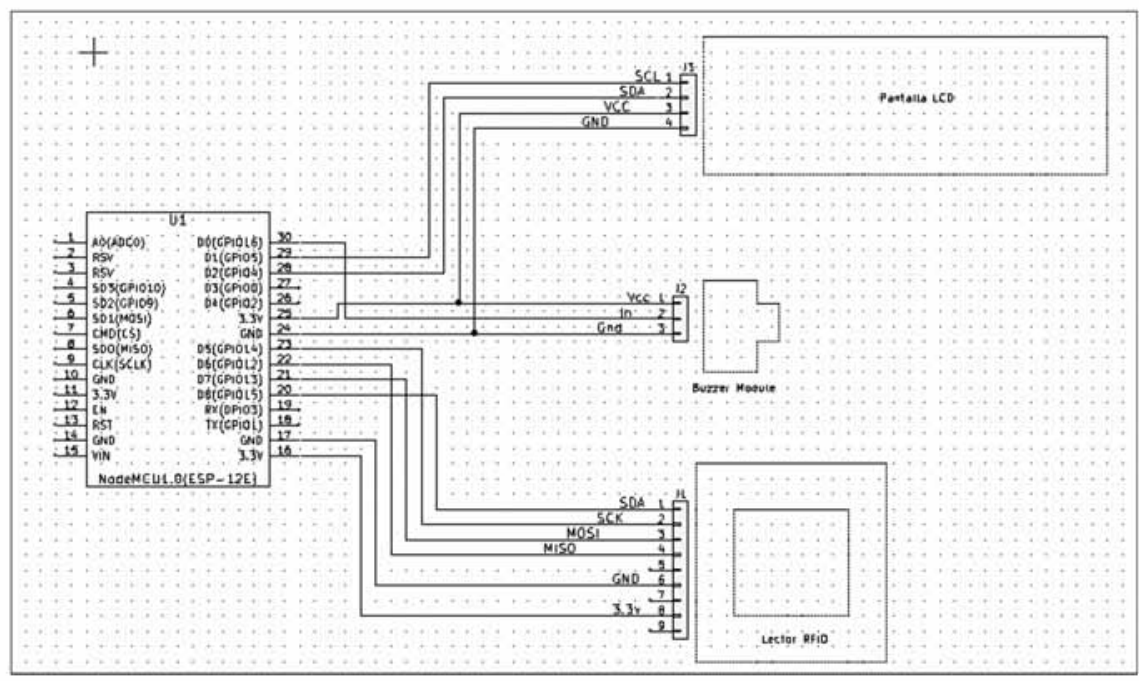

Fuente propia

Figura 3. Implementación del circuito electrónico de lectura.
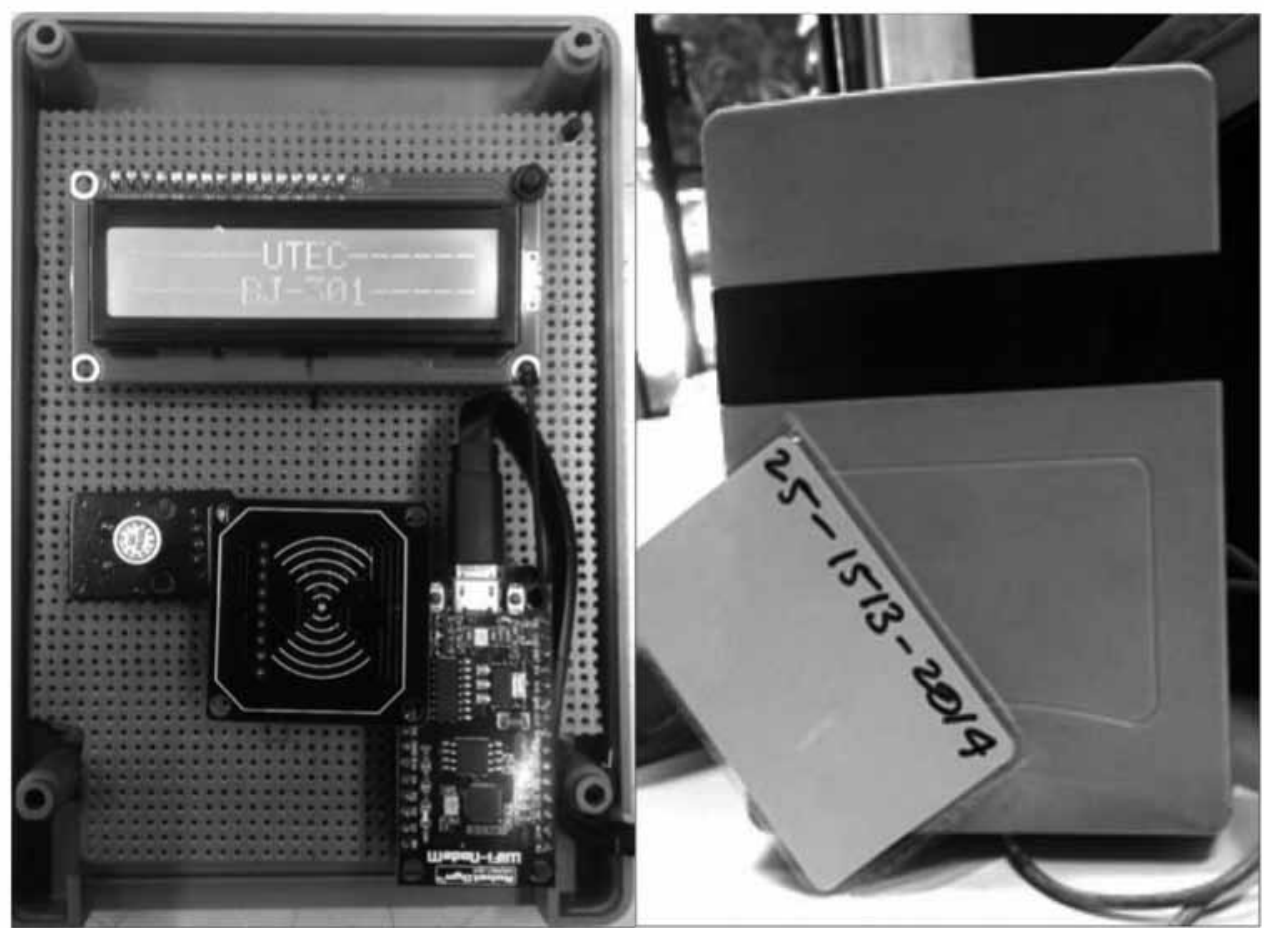

Fuente propia 


\section{Aplicación loT y visualización de datos}

En este bloque del sistema, que manejará la conexión entre el sensor electrónico y la etapa de presentación, se hizo uso de los servicios de Google App Script junto con Google Sheets. En la etapa de visualización se utilizó la herramienta de Google Sites para montar un sitio web para tener acceso desde un navegador visitando https://sites.google.com/a/mail.utec.edu. sv/labeleutec/bj301

Figura 4. Capturas del sitio para visualización de datos.

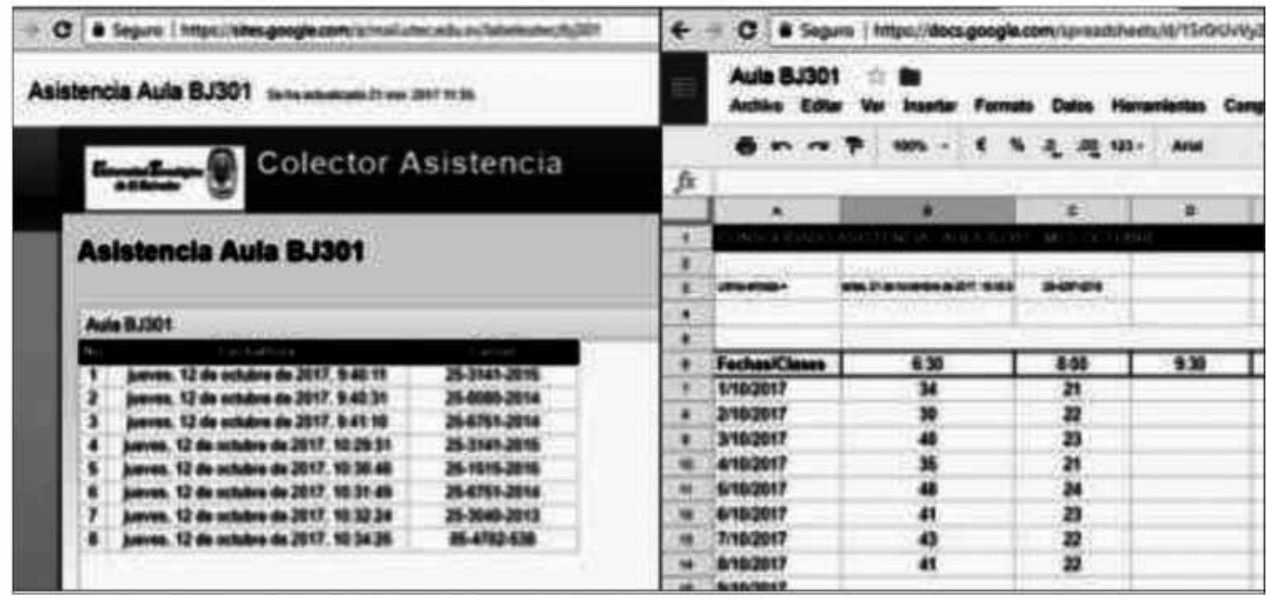

Fuente propia

\section{Conclusiones}

El sistema de IOT diseñado para el registro automatizado de las personas que ingresan a un aula cumple con el objetivo general planteado; y se convierte en una herramienta tecnológica economica que sirve de apoyo en las labores logísticas de una institución donde el registro de asistentes sea una tarea grande, periódica y muy importante dentro del quehacer administrativo del centro.

Con esta investigación se ha podido aportar nuevo conocimiento científico, de manera que se ha mostrado una nueva forma de hacer un sistema de IoT eficiente para la solución de problemas de automatización, monitoreo y control remoto de procesos, con herramientas tecnológicas recientes, de bajo costo y eficientes, tales como el microcontrolador ESP8266 y la plataforma Google, accesibles en el entorno local. El uso de los componentes mencionados permitió el diseño y construcción de un circuito electrónico embebido, que cumple con la función de permitir el escaneo de una tarjeta RFID, leer su información interna única, decodificarla y enviar vía Wi-Fi a internet. Este circuito además es de fácil configuración y reproducción, para producirlo e implementarlo masivamente, por ejemplo, en un campus.
El equipo de trabajo propone que se pueda tomar como base el conocimiento científico técnico aportado por este trabajo en el desarrollo de sistemas IoT, como el planteado, en instituciones similares a la Utec. Específicamente, se recomienda realizar el trabajo con el apoyo del Ministerio de Educación, Ciencia y Tecnología; y con posibles alianzas con sus centros educativos a escala nacional, para la puesta en marcha de aplicaciones como fruto de esta investigación.

\section{Referencias}

Grupo Ejje. (2017). "Hardware". Recuperado de http://www. ejje.com/hardware/

Kubitza, T., Voit, A., Weber, D., \& Schmidt, A. (2016). "An IOT infrastructure for ubiquitous notifications in intelligent living environments". International Joint Conference on Pervasive and Ubiquitous Computing Adjunct UbiComp. DOI: 10.1145/2968219.2968545.

Mcpherson, B. (2016). Going gas: from VBA to google apps script. California: O'Reilly Media

Sánchez, J.C. (2006). Metodología de la investigación científica y tecnológica. Madrid: Ediciones Díaz de Santos.

Tollervey, N. (2017). Programming with micropython. California: O'Reilly Media. 